REGARDS

SUR L'ECONOMIE ALLEMAND

BULLETIN ECONOMIQUE DU CIRAC
Regards sur l'économie allemande

Bulletin économique du CIRAC

$111 \mid 2013$

Varia

\title{
Un « modèle » se doit d'être exemplaire
}

Isabelle Bourgeois

\section{OpenEdition}

Journals

Édition électronique

URL : http://journals.openedition.org/rea/4623

DOI : $10.4000 /$ rea.4623

ISSN : 1965-0787

Éditeur

CIRAC

Édition imprimée

Date de publication : 1 décembre 2013

Pagination : 3-6

ISSN : 1156-8992

\section{Référence électronique}

Isabelle Bourgeois, «Un « modèle » se doit d'être exemplaire », Regards sur l'économie allemande [En ligne], 111 | décembre 2013, mis en ligne le 17 décembre 2013, consulté le 15 septembre 2020. URL http://journals.openedition.org/rea/4623 


\section{Un " modèle " se doit d'être exemplaire}

L'économie allemande fait preuve d'une santé solide. Certes, l'excédent commercial s'amenuise sous l'effet d'un tassement de la demande à la fois des pays industrialisés et des économies de la zone Euro, ce qui ralentit quelque peu la production industrielle et incite depuis plusieurs mois les entreprises à accroître leur taux d'utilisation des capacités plutôt qu'à investir. Mais ce recul est largement compensé par la demande intérieure qui tire seule la conjoncture depuis un an maintenant. Les ménages, rassurés par un marché du travail dont la situation frise le plein emploi et rendus confiants dans l'avenir par l'évolution de leur pouvoir d'achat, se montrent d'humeur à consommer. L'immobilier profite de la forte hausse de la demande de logements urbains comme de la faiblesse des taux d'intérêts qui incite les Allemands à placer leur épargne dans la pierre plutôt que dans les produits financiers.

$\mathrm{Au}$ total, les fondamentaux de l'économie allemande sont robustes. Avec la reprise qui s'esquisse en cette fin d'année dans les économies industrielles et le frémissement perceptible au sein d'une zone Euro relativement sereine depuis l'accalmie de la crise, les conjoncturistes se montrent optimistes. Dès le début de 2014, la croissance du PIB allemand devrait renouer avec un rythme de croisière de 1,6\%, voire plus. Dans ce contexte porteur, la consolidation budgétaire devrait se poursuivre : le déficit sera nul, et la dette pourra être progressivement réduite.

\begin{tabular}{|c|c|c|c|c|}
\hline \multicolumn{5}{|c|}{ Prévisions du Conseil des Sages } \\
\hline & 2011 & 2012 & $2013 P$ & 2014 P \\
\hline \multicolumn{5}{|l|}{ évolution } \\
\hline PIB (\%) & 3,3 & 0,7 & 0,4 & 1,6 \\
\hline Consommation (\%) & 2,0 & 0,8 & 1,0 & 1,3 \\
\hline privée (\%) & 2,3 & 0,8 & 1,0 & 1,4 \\
\hline publique (\%) & 1,0 & 1,0 & 0,9 & 1,1 \\
\hline \multicolumn{5}{|l|}{ Investissements } \\
\hline biens d'équipement (\%) & 5,8 & $-4,0$ & $-2,6$ & 6,2 \\
\hline construction (\%) & 7,8 & $-1,4$ & $-0,2$ & 4,1 \\
\hline autres (\%) & 5,1 & 3,4 & 3,0 & 4,7 \\
\hline Demande intérieure (\%) & 2,8 & $-0,3$ & 0,8 & 2,0 \\
\hline Part de l'excédent commercial dans le PIB (points de pourcentage) & 0,7 & 0,9 & $-0,3$ & $-0,2$ \\
\hline exportations (\%) & 8,0 & 3,2 & 0,2 & 5,2 \\
\hline importations (\%) & 7,4 & 1,4 & 1,0 & 6,3 \\
\hline Productivité horaire (\%) & 1,8 & 0,5 & 0,1 & 0,9 \\
\hline Coûts salariaux horaires (\%) & 1,0 & 3,1 & 2,4 & 1,5 \\
\hline Prix à la consommation (\%) & 2,1 & 2,0 & 1,5 & 1,9 \\
\hline Revenu disponible des ménages (\%) & 3,8 & 2,3 & 2,3 & 3,4 \\
\hline \multicolumn{5}{|l|}{ état } \\
\hline Actifs occupés (milliers) & 41152 & 41608 & 41860 & 42109 \\
\hline Actifs occupant un emploi soumis à cotisations sociales (milliers) & 28440 & 28991 & 29367 & 29679 \\
\hline Actifs occupant un «mini-job» a) & 7379 & 7397 & 7455 & 7481 \\
\hline Chômeurs indemnisés (milliers) ${ }^{b)}$ & 2976 & 2897 & 2954 & 2950 \\
\hline Taux de chômage (Agence fédérale pour l'emploi) (\%) & 7,1 & 6,8 & 6,9 & 6,8 \\
\hline Taux de chômage (critères OIT) (\%) & 5,7 & 5,3 & 5,3 & 5,2 \\
\hline Déficit public (\% du PIB) & $-0,8$ & 0,1 & 0,1 & 0,0 \\
\hline Dette publique $(\% d u P I B)^{c}$ & 80,0 & 81,0 & 78,3 & 75,0 \\
\hline
\end{tabular}

Source : Sachverständigenrat zur Begutachtung der gesamtwirtschaftlichen Entwicklung, Jahresgutachten 2013/14 (13-11-2013). NB : PIB et composantes en données corrigées des prix. a) plafond de $400 €$ par mois jusqu'en 2012 , de $450 €$ par mois depuis 2013 ; b) données de l'Agence fédérale pour l'emploi (Nuremberg); c) critères du Traité de Maastricht.

Après un hiver 2012/13 où les conditions météorologiques avaient été particulièrement défavorables à l'activité, celle-ci avait connu au deuxième trimestre 2013 un reElle est tirée par gain de dynamisme (+0,7\% par rapport au trimestre précédent ou $+0,9 \%$ par rapport à la même période de 2012). Après cette phase de rattrapage, l'activité s'est normalisée au troisième trimestre $(+0,3 \%$ par rapport au précédent ou $+1,1 \%$ par rapport au troisième trimestre 2012 ; Destatis). De juillet à septembre, la croissance du PIB était due exclusivement à la consommation intérieure. En effet, l'investissement a redémarré tant en ce qui concerne les biens d'équipement $(+1,0 \%$ en un an) que la cons-

L'économie allemande renoue avec son rythme de croisière

la consommation 
De belles perspectives ...

... mais le projet de la Grande coalition présente des risques

Un peu plus de redistribution

La consolidation budgétaire reste néanmoins prioritaire l'avenir de l'Allemagne" truction (+2,2\%). Les dépenses des ménages ont augmenté de 1,5\%, les dépenses publiques de $0,4 \%$. Quant à la balance commerciale, elle a été déficitaire (importations : $+1,9 \%$; exportations : $+0,7 \%$ ). Elle a tiré la croissance vers le bas d'un demi point de pourcentage.

Le retour de la croissance après la profonde récession de 2009 avait été interrompu en Allemagne par l'aggravation de la crise de la dette souveraine au sein de la zone Euro. Dans la situation de plus grande sérénité actuelle, il regagne en force, porté par la confiance des acteurs économiques, comme en attestent à l'unisson tous les indices du climat des affaires ou du moral des ménages. Et " avec la poursuite de l'embellie conjoncturelle en cours dans les pays industrialisés et l'amélioration de la conjoncture dans la zone Euro, les exportations et, à leur suite, l'investissement des entreprises comme les importations devraient renouer avec la hausse ", résume la Bundesbank dans ses prévisions du 6 décembre.

C'est sur cette toile de fond et ses perspectives favorables que se sont engagées les négociations entre l'Union CDU/CSU et le SPD pour constituer un gouvernement fédéral de Grande coalition. Elles n'étaient pas encore achevées au moment où le groupe de travail des Instituts de conjoncture publiait son rapport conjoncturel d'automne (le 17 octobre), et où le Conseil des Sages remettait son rapport annuel au gouvernement sortant (le 13 novembre). Le contrat de coalition n'a en effet été signé que le 27 novembre.

Instituts et Conseil des Sages fondent donc leurs prévisions sur le statu quo en matière de politique économique nationale, ne cachant pas qu'elles sont entachées d'un certain nombre de risques. En externe, la crise de la dette souveraine au sein de la zone Euro est loin d'être résolue, et les problèmes structurels de compétitivité sont autant de foyers de crise potentiels. En interne, le projet du nouveau gouvernement comporte un certain nombre de mesures potentiellement dommageables à la compétitivité économique et à la cohésion sociale.

\section{Le projet de politique économique et sociale du contrat de coalition}

"Configurer Intitulé «Configurer l'avenir de l'Allemagne» (Deutschlands Zukunft gestalten), le contrat de coalition, un document de 185 pages, présente les lignes directrices de la future politique gouvernementale. Ce projet - un compromis - est le fruit d'un savant dosage entre les thèmes clés des programmes électoraux des partis constitutifs de la Grande coalition. Les sept axes développés ne sont encore qu'une 'feuille de route' : en effet, "les partenaires de la coalition s'engagent à mettre en œuvre cet accord dans leur politique gouvernementale ", ainsi qu'ils le rappellent. Entre les objectifs affichés et les dispositions concrètes des lois qui seront effectivement adoptées au fil de la législature, il faut donc s'attendre à un certain nombre d'ajustements. Cela vaut particulièrement pour les points les plus vivement débattus actuellement dans l'espace public, dont l'introduction d'un salaire minimum légal généralisé. Car la définition de la politique gouvernementale allemande s'effectue toujours collectivement via une large discussion avec la société civile qui fait valoir ses intérêts et ses choix non seulement par l'acte de vote, mais aussi, une fois les majorités établies, par sa contribution active au débat public.

Dès le préambule, le ton est donné : “L’Allemagne a connu, ces dernières années, un essor économique comme nul autre pays dans l'UE ", grâce à des réformes structurelles et à l'action de tous, politique et société, en premier lieu partenaires sociaux. "Mais tous n'ont pas pu participer à cette évolution positive », et durant les quatre années à venir, "nous voulons poursuivre sur cette voie de progrès et surmonter les anomalies existantes ". II s'agit donc de redistribuer mieux les richesses et d'accroître la participation. Sans toutefois remettre en question la doctrine sur laquelle repose le modèle économique allemand ni la responsabilité de l'Allemagne au sein de l'UE: "Nous voulons conforter l'économie sociale de marché, ancrer ses principes en Europe et par-delà, et veiller à créer des conditions-cadre pour que notre pays offre à tous et à chacun un accès équitable à un bel avenir ».

Après ces déclarations d'intention sont énumérées les lignes directrices de la future politique. En tête figure : "renoncer à tout nouvel endettement et réduire le taux de la dette », suivie de "développer la compétitivité et accroître les investissements » et définir "des règles pour les marchés financiers pour protéger contribuables et épar- 
gnants ». La politique budgétaire et la politique européenne de l'Allemagne sont donc inscrites dans la continuité. Ce n'est qu'en quatrième position que figure la revendication électorale clé du SPD : "Introduire un salaire minimum, prévenir l'abus en matière de contrats d'entreprise et de travail intérimaire ", suivie de : "Accroître l'équité des chances par la formation et la qualification ». Ensuite, sont énumérés divers objectifs : investissement dans les infrastructures, changement énergétique, extension du haut débit, réforme du fédéralisme financier, hausse du minimum vieillesse, engagement dans la poursuite de l'approfondissement de l'Europe et politique de paix. Ce programme (voir dans ce numéro l'analyse détaillée de $\mathrm{H}$. Uterwedde) reste fidèle somme toute aux principes de l'économie sociale de marché : avant de redistribuer les richesses, il faut les créer, donc améliorer le cadre des activités. Et il confirme explicitement l'ancrage de la politique allemande dans l'Europe.

Ces grandes orientations sont ensuite détaillées en sept chapitres dans le corps du contrat : "Croissance, innovation et prospérité », "Plein emploi, travail de qualité et sécurité sociale ", "Des finances robustes ", "Cohésion de la société ", "Etat moderne, sécurité intérieure et droits des citoyens », "Une Europe puissante », "Responsabilité [de l'Allemagne] dans le monde ».

Les axes les plus largement discutés dans l'espace public sont au nombre de trois. Comment financer l'investissement dans les infrastructures de transport, le système de formation et les structures d'accueil de la petite enfance ? Le contrat de coalition écarte toute hausse des impôts tout en mettant l'accent sur la poursuite de la consolidation budgétaire. A cela s'ajoute la question du financement des énergies renouvelables et des infrastructures de transport de l'électricité dans le contexte de la sortie du nucléaire. Le deuxième grand thème du débat concerne la réorientation du régime des retraites vers plus de redistribution, alors même que son financement n'est garanti que jusqu'à l'horizon 2030 tout au plus. Enfin, le thème le plus vivement controversé est celui de l'introduction d'un SMIC légal généralisé de 8,50 € en 2017.

\section{L'évaluation des experts}

Dans la partie de leur rapport consacré à l'évaluation des politiques, les Instituts passent en revue certains projets gouvernementaux qui, "s'ils étaient mis en œuvre, seraient susceptibles de modifier l'orientation des politiques économique, budgétaire et sociale ». Ils soulignent, sans trouver à y redire, que les inflexions esquissées poursuivent deux objectifs : la redistribution des richesses et le soutien de la croissance. Les mesures envisagées (confirmées depuis par le contrat de coalition) sont en effet établies sur le constat de finances publiques en voie de consolidation : pour la première fois depuis 2000 , le budget allemand était à l'équilibre en 2012 ; il le sera également en 2013. Or, critiquent les Instituts, cet état résulte plus d'une conjonction de facteurs favorables que d'une véritable politique de réduction des dépenses publiques : taux d'intérêts historiquement faibles pour les obligations d'Etat, hausse des recettes fiscales sous l'effet de la levée du gel du barème de l'impôt sur le revenu en 2010 et de la bonne tenue du marché de l'emploi comme de l'activité.

Un cap de consolidation budgétaire plus rigoureux est donc impératif, ne serait-ce que pour préserver la latitude d'action du prochain gouvernement en matière d'investissement et de « justice sociale ». Et pour mieux lui montrer la voie - mais aussi les limites à ne pas franchir -, les Instituts calculent le montant de cette marge de manœuvre. II s'élèverait à 33 milliards $€$ d'ici 2018 , ce qui représente une 'cagnotte' suffisante pour financer dépenses et investissements supplémentaires sans augmenter les impôts, concluent-ils. Ils semblent avoir été entendus : le projet d'impôt sur la fortune, cher au SPD mais très controversé, a été rejeté. Il aurait lourdement taxé le patrimoine des entreprises du Mittelstand (majoritairement des familiales) et détruit des emplois, ce qui risquait de tasser la croissance et de peser sur l'encours fiscal.

Rappelant que la relative bonne santé des finances publiques est largement imputable à la robustesse du marché de l'emploi et que la situation actuelle de quasi plein emploi est le résultat d'une politique conséquente de flexibilisation du marché du travail menée par les partenaires sociaux depuis le début du millénaire, suivie des réformes adoptées sous le chancelier Schröder, les Instituts tordent le cou aux arguments de campagne électorale selon lesquels la qualité du travail se serait lourdement dégradée (voir REA 110/2013). Non seulement on n'observe pas de précarisation ou de paupérisation généralisées, mais les emplois créés sont majoritairement
Trois chantiers politiques hautement controversés

Les Instituts estiment la marge de manœuvre à 33 milliards $€$

Le SMIC légal aura des effets désastreux 
Le Conseil des Sages " contre une politique tournée vers le passé " des emplois réguliers, et les montants des salaires effectivement versés sont supérieurs à ceux des salaires conventionnels de branche.

Cela placé, les Instituts partent en guerre contre l'introduction d'un SMIC légal généralisé : un tel salaire, " uniforme, c'est-à-dire s'appliquant pareillement à toutes les branches et à toutes les régions, aurait assurément des effets nettement plus négatifs sur le marché de l'emploi » que les quelques SMIC sectoriels adoptés jusqu'ici. Ses effets seraient désastreux surtout dans l'est de l'Allemagne, où un quart des actifs est rémunéré en dessous de 8,50 € l'heure, de même qu'il générerait une vague de licenciements économiques dans le tissu des petites PME. Enfin, "il faut se garder de surestimer l'impact d'un tel SMIC légal sur la distribution des revenus » et donc la consommation, insistent-ils.

Le Conseil des Sages entre plus avant dans l'analyse. Sa critique du programme gouvernemental est cinglante, l'amenant à intituler son rapport annuel : "Contre une politique économique tournée vers le passé ». S'il démonte lui aussi le projet d'un SMIC légal, son principal grief est le suivant: "Le contexte économique actuel et la bonne position de l'Allemagne en comparaison des pays en crise dans la zone Euro semblent avoir très largement fait perdre de vue les grands défis futurs ». II incrimine le fait que nombre de mesures envisagées, comme un supplément de pension de retraite versé aux mères d'enfants nés avant 1992, la hausse du minimum vieillesse allemand (qui serait porté à $850 €$ et financé par l'impôt), la multiplication des régimes d'exception à l'âge légal de départ à la retraite (67 ans), ne sont pas soutenables et pénalisent les générations futures.

Plus fondamentalement encore, le Conseil souligne que "les défis du futur seront d'autant plus difficiles à relever que les réformes de l'Agenda 2010 auront été diluées ou partiellement remises à plat », avant de poursuivre : "le même constat s'applique aux mesures dommageables à la croissance et à l'emploi comme le SMIC ou des hausses d'impôt». Bien au contraire, il convient de poursuivre sur la voie des réformes pour créer un environnement encore plus favorable à l'activité. "Une telle politique orientée vers l'avenir est nécessaire pour mieux asseoir la croissance allemande face au vieillissement démographique à venir et pour assurer la soutenabilité des budgets publics comme des systèmes de protection sociale 》.

Rappelons que cette haute autorité indépendante de l'Etat, créée en 1967 (sous la première Grande coalition), a pour mission expresse d'évaluer les politiques gouvernementales et de formuler des recommandations. Elle avait ainsi par exemple joué un rôle déterminant dans l'adoption des grandes réformes structurelles menées par le gouvernement Schröder II. Elle accompagnera aussi de sa critique constructive l'action de la nouvelle Grande coalition.

«RESPONSABILITÉ NATIONALE ET FONCTION DE MODĖLE ». La stabilité du modèle économique et social allemand dans l'UE implique de lourdes responsabilités, rappelle le Conseil des Sages. Et il souligne que si la gestion de crise de la BCE a permis de gagner du temps dans l'urgence, c'est aux politiques nationales qu'il revient de résoudre les problèmes de fond de l'UE. "Le gouvernement fédéral ne devrait pas créer l'impression d'attendre, voire d'exiger que d'autres pays engagent des processus d'adaptation douloureux, tout en renâclant à prendre des mesures impopulaires dans son propre pays ».

Au lieu de chercher à reréguler le marché du travail grâce à un SMIC ou autres mesures, il convient au contraire de poursuivre sa flexibilisation pour inclure les moins qualifiés. Au lieu de se contenter d'un budget à l'équilibre grâce à la croissance, il faut consolider les finances publiques en réduisant la dette. A cet effet, une réforme en profondeur du fédéralisme financier (voir REA 102/2011) s'impose, attribuant une plus grande responsabilité fiscale aux Länder. Le code fiscal est à réformer lui aussi. La politique énergétique doit être mieux coordonnée et intégrer le coût économique de la sortie du nucléaire. Le financement de l'assurance maladie et de l'assurance dépendance doit être pérennisé. Ces efforts domestiques sont d'autant plus incontournables que l'Allemagne est aujourd'hui l'un des grands architectes de l'approfondissement de l'UE. Sa responsabilité européenne de «modèle » oblige. Elle a pour contrepartie le devoir d'exemplarité.

I. Bourgeois (10-12-2013) 